\title{
Decrease in emergency department attendances during COVID-19 especially in school-going children
}

\section{Dear Editor,}

Health-seeking behaviour varies during a pandemic. Early reports have suggested reduced attendances at emergency departments (EDs), especially in paediatric patients and in patients with minor ailments, but these observations have yet to be evaluated in Singapore. ${ }^{1,2}$ We investigated ED attendances during the coronavirus disease 2019 (COVID-19) pandemic in Singapore.

A retrospective study comprising all patients who attended the general ED of a restructured tertiary hospital between 1 July 2019 and 30 June 2020 was carried out. Four time periods were identified: (1) before COVID-19 pandemic (1 July 2019 to 31 December 2019); (2) initial COVID-19 pandemic (1 January 2020 to 6 April 2020); (3) circuit breaker (7 April 2020 to 1 June 2020); and (4) after circuit breaker (2 June 2020 to 30 June 2020). The term "circuit breaker" refers to a period of stricter social distancing measures that included closure of most workplaces and full home-based learning for schools. In this letter, we report the time periods from initial COVID-19 pandemic, circuit breaker and after circuit breaker as during COVID-19 pandemic.

Information including demographics, referral source, mode of arrival, triage category, case type (trauma or non-trauma), Systematized Nomenclature of Medicine Clinical Terms used, disposition as well as mortality outcomes in the ED, were collected using a standardised form. This study was approved by SingHealth Institutional Review Board (CIRB reference 2020/2611). SPSS Statistics software version 25 (IBM Corp, Armonk, US) was used for analysis. Association between categorical variable was assessed using chi-square test. Comparison of medians was assessed using Mann-Whitney U test or Kruskal-Wallis test. Statistical significance was taken at $P<0.05$.

There were 105,256 ED attendances. The median daily attendance was lower during the pandemic than before. During the pandemic, the median daily attendance was highest during the initial phase, lowest during the circuit breaker, and lower than pre-pandemic numbers after the circuit breaker.

The number of attendances fell for every age group; however only paediatric patients $(<16$ years old) showed a significant decrease during the pandemic. A higher proportion of attendances to the ED were self-referrals, with a drop in referrals by primary care. Despite the increase in self-referrals, there was an increase in emergency medical services (EMS) ambulance use for conveyance to the ED (Table 1).

During the pandemic, the ED also saw a decrease in ambulatory (P3) cases. Interestingly, there was a gradual increase of ambulatory (P3) cases at the initial phase of the pandemic before the sharp decrease observed when circuit breaker was implemented. The proportion of trauma-related attendances also dropped significantly during the pandemic with the lowest recorded during the circuit breaker. Upper respiratory tract infection (URTI) remained the top diagnosis both before and during the pandemic, but the proportion almost doubled during the pandemic (Table 1).

The proportion of attendances requiring admission to the hospital was higher during the pandemic. However, the admissions to the intensive care, high dependency, and extended diagnostic and treatment units were not statistically different. Overall, the mortality remained low but significantly higher during the pandemic (Table 1).

As healthcare systems respond to the pandemic, EDs at the frontline of the healthcare response take on an evolving role. At the early phase of the pandemic in Singapore, performing confirmatory testing for COVID-19 was limited to EDs such that patients diagnosed with COVID-19 would be admitted for inpatient care through an ED. These additional responsibilities resulted in a change in health-seeking behaviour as evidenced by the increase in self-referrals to the ED and URTI cases. These patients, who would otherwise be seen at the primary care setting, presented at the ED instead due to the availability of confirmatory testing and the possible need for inpatient admission. Similarly, healthcare measures by the Ministry of Health, which included establishing a special ambulance service for transporting suspected cases of COVID-19, had resulted in an increase in patients turning to EMS ambulances for conveyance to the hospital, possibly to limit their interactions with the community.

ED attendance is not a direct reflection of the disease burden of COVID-19 on the population but rather a complex interplay of control measures established to limit contagion spread within the community, as well as healthcare measures implemented to diagnose and 


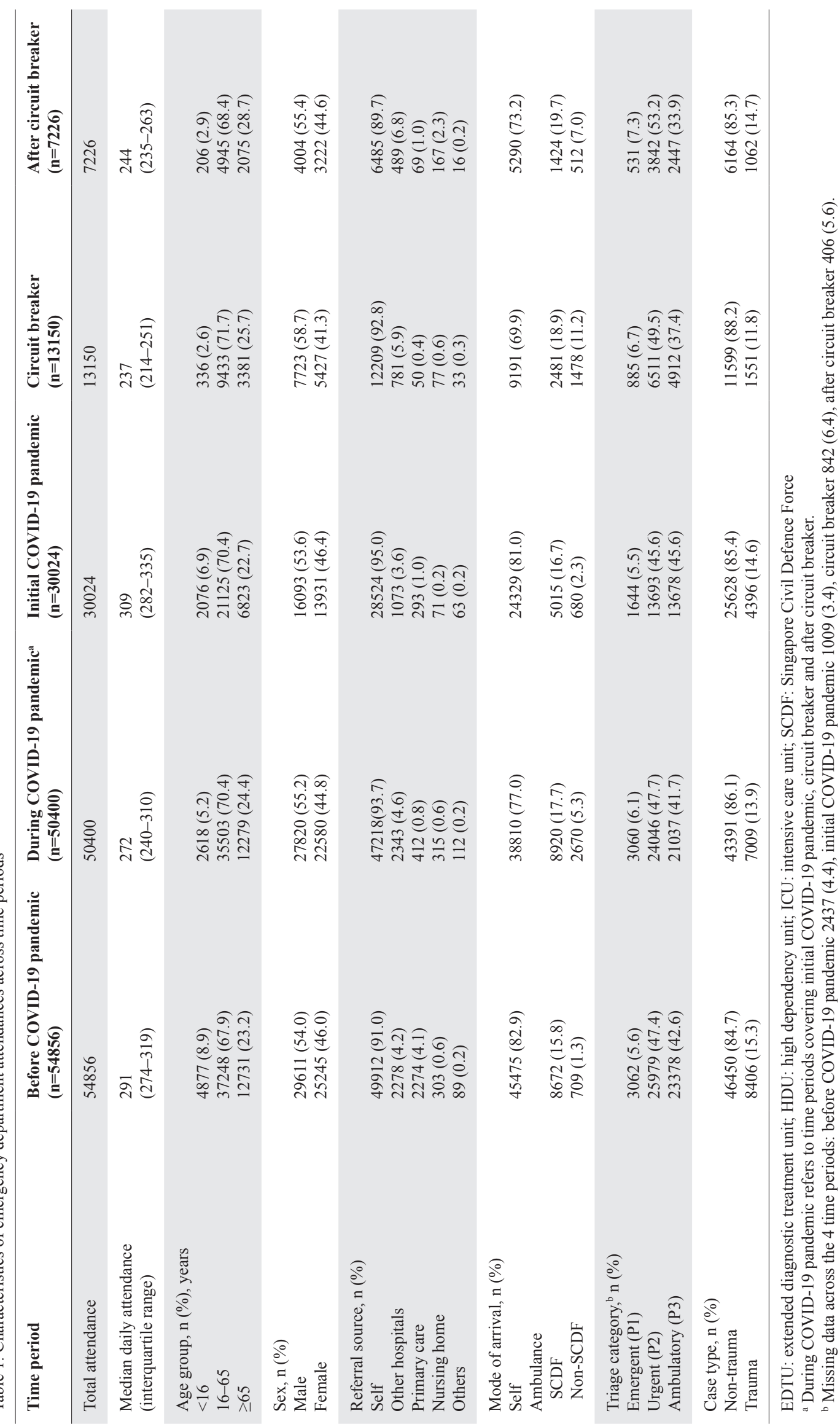




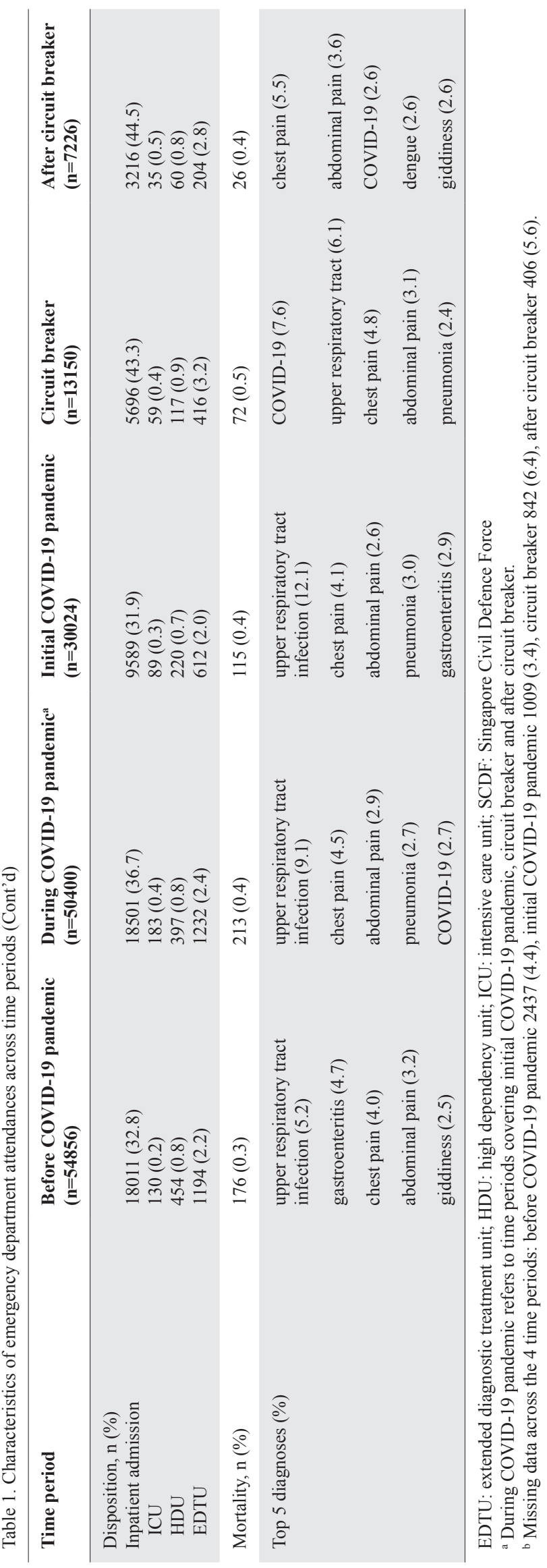

treat patients with COVID-19. The decrease in ED attendances was similar with trends observed by EDs internationally and in Singapore. ${ }^{2-8}$ While these trends may be attributable to patients keeping away from EDs due to the fear of exposure to COVID-19 patients, or people exercising civic responsibility by making a deliberate choice to avoid utilising EDs so that healthcare resources would not be stretched, ED attendances reached a nadir during the circuit breaker, suggesting that movement restrictions played a part in reducing ED visits. ${ }^{9}$ It is therefore important to ensure that emergency care remains accessible to patients despite control measures in place.

Healthcare authorities need to emphasise that patients should not delay ED visits for urgent and potentially serious medical conditions. On the other hand, guidance must also be provided for use of appropriate healthcare facilities and alternative resources for non-urgent conditions. The use of smartphone applications may allow patients to self-report their symptoms and be directed to healthcare facilities appropriate for their conditions, or to provide reassurance and advice for self-management and monitoring of any red-flag symptoms. ${ }^{10}$ Telemedicine using smartphones or webcam-enabled computers may be utilised for forward triage by screening patients who require emergency care, or to conduct medical consultations between patients and clinicians, thereby reducing ED attendances. ${ }^{11,12}$ Engaging primary care providers early will allow more clinics to be prepared and equipped to take on specialised roles in response to the pandemic. For instance, Public Health Preparedness Clinics attend to patients with URTI with clear guidelines for onward referral to EDs, while Swab and Send Home Clinics perform confirmatory testing for COVID-19. Both clinics are important as they represent the enhanced capacity and capability of Singapore's primary care response during the pandemic, thus preserving ED manpower and resources to attend to patients who are more unwell.

This was a retrospective study based on department census, as such it has the potential for missing information and misclassification. As a single-centre study, the observations may not be generalisable. The impact of changes in attendance on the institution or healthcare system, as well as their long-term consequences warrant further studies. As EDs are an integral part of the healthcare system during pandemics, this study will serve as a reference point for emergency care, as well as a starting point for further collaborative efforts between EDs at a national level. 


\section{REFERENCES}

1. Martín-Sánchez FJ, Valls Carbó A, López Picado A, et al. Impact of Spanish public health measures on emergency visits and COVID-19 diagnosed cases during the pandemic in Madrid. Rev Esp Quimioter 2020;33:274-7.

2. Hartnett KP, Kite-Powell A, DeVies J, et al. Impact of the COVID-19 pandemic on emergency department visits. MMWR Morb Mortal Wkly Rep 2020;69:699-704.

3. K Honeyford, Coughlan C, Nijman R, et al. Changes in emergency department attendances before and after COVID-19 lockdown implementation: a cross sectional study of one urban NHS Hospital Trust. medRxiv 2020. DOI: https://doi.org/10.1101/2020.07.20. 20157560

4. Leow SH, Dean W, Macdonald-Nethercott M, et al. The Attend study: a retrospective observational study of emergency department attendances during the early stages of the COVID-19 pandemic. Cureus 2020;12:e9328.

5. Walline JH, Song PP, Lim AM, et al. Hong Kong emergency department attendance plummets during COVID-19. Emerg Med Australas 2020;32:1093-4.

6. Roland D, Harwood R, Bishop N, et al. Children's emergency presentations during the COVID-19 pandemic. Lancet Child Adolesc Health 2020;4:e32-3.

7. Isba R, Edge R, Jenner R, et al. Where have all the children gone? Decreases in paediatric emergency department attendances at the start of the COVID-19 pandemic of 2020. Arch Dis Child 2020;105:704.

8. Tan RMR, Ganapathy S, Tyebally A, et al. Paediatric emergency department attendances in Singapore during COVID-19 and SARS. Ann Acad Med Singap 2021;50:126-34.

9. Rosenbaum L. The untold toll-the pandemic's effects on patients without Covid-19. N Engl J Med 2020;382:2368-71.

10. Verzantvoort NCM, Teunis T, Verheij TJM, et al. Self-triage for acute primary care via a smartphone application: Practical, safe and efficient? PLoS One 2018;13:e0199284.

11. Hollander JE, Carr BG. Virtually perfect? Telemedicine for COVID-19. N Engl J Med 2020;382:1679-81.

12. Wang LY, Low TT, Yeo TJ. Telehealth in COVID-19 and cardiovascular disease-ensuring equitable care. Ann Acad Med Singap 2020;49:902-4.

Hannah Hui En Ang, ${ }^{1}$ MMED (Emerg Med),

Eunizar Omar, ${ }^{1}$ MRCEM, Jen Heng Pek, ${ }^{1}$ MMED (Emerg Med)

${ }^{1}$ Department of Emergency Medicine, Sengkang General Hospital, Singapore

Correspondence: Dr Jen Heng Pek, Department of Emergency Medicine, Sengkang General Hospital, 110 Sengkang E Way, Singapore 544886. Email: pek.jen.heng@singhealth.com.sg 